Psychotherapeut 2022 $67: 150-157$ https://doi.org/10.1007/s00278-021-00568-5 Angenommen: 30. November 2021 Online publiziert: 18. Januar 2022

○ Der/die Autor(en) 2022

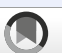

Check for
updates

\section{Sprachliche Charakteristika einer Psychotherapiesitzungssequenz aus der Perspektive unterschiedlicher Analysemethoden}

\author{
Antje Gumz ${ }^{1,2} \cdot$ Thomas Spranz-Fogasy ${ }^{3}$ \\ ' Professur für Psychosomatik und Psychotherapie, Psychologische Hochschule Berlin, Berlin, Deutschland \\ ${ }^{2}$ Klinik für Psychosomatische Medizin und Psychotherapie, Universitätsklinikum Hamburg-Eppendorf, \\ Hamburg, Deutschland \\ ${ }^{3}$ Leibniz-Institut für Deutsche Sprache, Mannheim, Deutschland
}

Zusammenfassung

\section{In diesem Beitrag}

- Ziele der Arbeit

- Material und Methoden

Fokus Therapeutenäußerungen - Psychodynamische Interventionsliste - Fokus Dialog - Konversationsanalyse • Sitzungsausschnitt • Transkription des Sitzungsausschnitts

- Ergebnisse Sitzungsausschnitt aus Perspektive der psychodynamischen Theorie - Mikroanalyse Fokus Therapeutenäußerungen Psychodynamische Interventionsliste • Mikroanalyse Fokus Dialog - Konversationsanalyse

- Diskussion

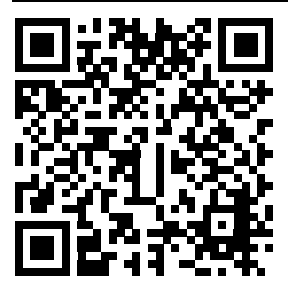

QR-Code scannen \& Beitrag online lesen
Hintergrund: Die sprachlichen Äußerungen sind ein zentrales Medium in Psychotherapien, d.h., Psychotherapie wirkt im Wesentlichen über die Sprache, über das Miteinanderreden. Angesichts der Bedeutung des sprachlichen Austauschs ist es relevant, die Mechanismen, über die Sprache in Psychotherapieprozessen wirkt, genauer zu verstehen. Die linguistische Psychotherapieforschung nutzt hierfür vielfältige Methoden.

Ziel der Arbeit: Vorliegender Beitrag demonstriert exemplarisch 2 mikroanalytische Ansätze.

Material und Methoden: Eine transkribierte Psychotherapiesitzungssequenz wurde aus Perspektive der psychodynamischen Theorie inhaltlich interpretiert und bezüglich sprachlicher Merkmale mithilfe von 2 Methoden mikroanalytisch beurteilt: Die verbalen Techniken (Fokus Therapeutenäußerungen) wurden mithilfe der Psychodynamischen Interventionsliste (PIL) geratet und eine detaillierte Konversationsanalyse (Fokus Dialog) erfolgte.

Ergebnisse: Analysen mit der PIL zeigten, dass im Sitzungsausschnitt überwiegend die Techniken „Bedeutung hinzufügen“ und „Wiederholen, Umschreiben, Zusammenfassen" verwendet wurden. Thematisch wurde besonders auf den „Vater" Bezug genommen, gefolgt von der "Therapeutin“. Der zeitliche Bezug lag schwerpunktmäßig in der "Vergangenheit". Die Gesprächsanalyse rekonstruiert, dass der Wechsel auf die Erlebensebene die Therapiesituation selbst in den Fokus rückt. Mithilfe sequenzieller Handlungszwänge werden extratherapeutische Konstellationen in der Vergangenheit und therapeutische Gegenwart kontrastierbar sowie intersubjektiv bearbeitbar gemacht.

Schlussfolgerung: Die eigene Sprache und den Dialog im Therapieprozess zu beobachten, kann für Therapeuten aufschlussreiche Erkenntnisse über Folgen und Voraussetzungen eigener Interventionen liefern. Forschungen an der interdisziplinären Schnittstelle von Psychotherapie und Linguistik sind lohnenswert.

\section{Schlüsselwörter}

Linguistik - Verbales Verhalten · Psychotherapeutische Prozesse · Psychodynamische Interventionsliste $\cdot$ Sequenzanalyse 
Die Sprache ist das Werkzeug des Psychotherapeuten. Therapeuten intervenieren primär verbal, und der sprachliche Austausch, d.h. die verbalen ÄuBerungen, machen einen bedeutsamen Teil der Aktivitäten und Geschehnisse in den Sitzungen aus. Es liegt daher nahe, besonderes Augenmerk auf die Sprache, auf den verbalen Dialog zwischen Patient und Therapeut zu richten und zu erforschen, wie verbale Techniken und sprachliche Merkmale der Interaktion zum therapeutischen Fortschritt beitragen.

\section{Hintergrund}

\section{Durch Worte heilen}

Psychotherapie gilt traditionell als „talking cure" (Breuer und Freud 2007 [1895]), d. h. als Heilverfahren, das im Wesentlichen über die Sprache, das Miteinanderreden wirkt (Marx et al. 2021, 2017). Die sprachlichen Äußerungen sind zentrales Medium in Psychotherapien. Auch wenn die nonverbale therapeutische Kommunikation äußerst bedeutsam und mit verbalen Informationen und prosodischen Merkmalen eng verflochten ist, intervenieren Therapeuten dennoch primär verbal, und die verbalen Äußerungen machen einen bedeutsamen Teil der Aktivitäten und Geschehnisse in den Sitzungen aus (Gumz et al. 2015).

Angesichts der Bedeutung des sprachlichen Austauschs in der Psychotherapie ist es relevant, die Mechanismen, über die das Miteinanderreden wirkt, genauer zu verstehen.

\section{Betrachtung sprachlicher Merkmale in der Psychotherapieforschung}

Die linguistische Psychotherapieforschung ist methodisch vielfältig. Datenbasis sind die verbalen Äußerungen, meist in Form von Transkripten.

Der Auflösungsgrad, mit dem der therapeutische Dialog oder die therapeutischen Äußerungen wissenschaftlich betrachtet wird/werden, ist unterschiedlich. Es gibt Messinstrumente, mit denen verbale Techniken global erfasst werden (Raten kompletter Sitzungen), und Methoden, bei denen einzelne Äußerungen, Sätze oder
Sprecherwechsel mikroanalytisch betrachtet werden (Gumz et al. 2015; Deppermann 2008). Globale Messungen sind zeitsparender und ermöglichen die Betrachtung einer größeren Stichprobe. Gleichzeitig sind sie anfälliger für kognitive Verzerrungen und erlauben nur eine grobe Erfassung der komplexen Prozesse (Levitt et al. 2017). Mikroanalysen verbaler, nonverbaler und interaktionaler Einheiten unterschiedlicher Größenordnung dagegen sind sehr zeitaufwendig, wodurch die analysierbare Datenmenge gering und es nur begrenzt möglich ist, die Ergebnisse zu generalisieren. Oftmals sind nur Einzelfallanalysen möglich. Jedoch ermöglicht eine feinauflösende Analyseebene, das verbale Geschehen detailliert zu beobachten und die Prozesse, die zu therapeutischer Veränderung führen, ausführlich zu erklären (Gumzetal. 2015).Grundlegend lassen sich zwei methodische Ansätze unterscheiden:

Quantitative Methoden zielen darauf $\mathrm{ab}$, sprachliche Merkmale metrisch oder kategorial zu erfassen und den Zusammenhang der Merkmale mit dem Therapieergebnis, der Sitzungsqualität (Mikro-Outcome) oder anderen relevanten Prozessvariablen zu analysieren. Hierfür wurde eine Reihe von Messinstrumenten entwickelt (Steinert et al. 2022; Gumz et al. 2015), die auf unterschiedliche Forschungsfragen zugeschnitten sind. Die Analysen können beobachterbasiert oder computerautomatisiert erfolgen. Betrachtet werden unterschiedliche sprachliche Merkmale, beispielsweise Wortkonnotationen wie die emotionale oder kognitive Färbung, Symbolisierungen, Metaphern oder Narrative oder auch verbale Techniken, wie Deutungen oder Selbstoffenbarungen des Therapeuten. Computergestützte Analysemethoden gehen meist deduktiv vor, suchen automatisiert nach Wörtern einer Kategorie und berechnen ihre relative Häufigkeit (Steinert et al. 2022; Marx et al. 2017).

Die Befundlage zu Forschungen zum Zusammenhang von Sprache mit dem Therapieergebnis oder anderen Prozessvariablen ist insgesamt noch schwach. Die ersten vorliegenden Studien deuten beispielsweise darauf hin, dass eine stärker emotional konnotierte und weniger kognitive bzw. affektiv neutrale Sprache der Therapeuten sowie eine den Patienten raumgebende und gleichzeitig engagierte Haltung mit einem besseren Therapieergebnis assoziiert ist (Steinert et al. 2022). Supportive und explorative verbale Techniken sowie das Ansprechen von Merkmalen der therapeutischen Beziehung scheinen einen positiven Effekt zu haben, kontrollierende und herausfordernde Äußerungen dagegen einen negativen (Kadur et al. 2020). Zudem scheint das "connecting" den Therapieerfolg zu fördern (auf linguistischer Textanalyse basierendes Maß für den inneren Zugang des Patienten zu Gefühlen mit der gleichzeitigen Fähigkeit, darüber zu reflektieren; Gelo 2022).

Qualitative Methoden zielen daraufab, linguistische Merkmale, d.h. die sprachlichen Prozesse von Psychotherapie sehr feinauflösend zu beschreiben. Hier existieren wiederum unterschiedliche methodische Paradigmen, wie die linguistische Gesprächsanalyse, die narrative Analyse, die Diskursanalyse (Marx et al. 2017; Sidnell und Stivers 2013; Georgaca und Avdi 2009).

Unabhängig von der Analysemethode kann man sprachliche Merkmale von Psychotherapiesitzungen aus zwei Perspektiven betrachten. Der Fokus kann auf dem therapeutischen Dialog oder spezifisch auf den sprachlichen Äußerungen des Patienten oder Therapeuten liegen (• Abb. 1).

Für die sprachlichen Äußerungen des Therapeuten werden verschiedene Begriffe synonym verwendet, wie "verbal response mode", "verbal statements" (Kadur et al. 2020), „,verbal interactions“, „,verbale Interventionen" oder "verbale Techniken" (Gumz 2018; Gumz et al. 2017, 2015). Die verbalen Techniken enthalten konkret beobachtbare und latente Merkmale. Konkrete Merkmale beziehen sich auf den semantischen Inhalt der Äußerungen („Was wurde gesagt?"). Latente Merkmale beschreiben den impliziten pragmatischen Gehalt einer Äußerung („Was ist gemeint oder implizit enthalten?"). Beispiele für latente Merkmale sind qualitative Charakteristiken (z. B. Wärme oder Kompetenz des Therapeuten), therapeutische Haltungen (z. B. Abstinenz) oder Intentionen (z. B. den Dialog lenken oder Affekte kontrollieren). Bei den konkreten Merkmalen lassen sich drei Beschreibungsdimensionen differenzieren: a) formale Merkmale (z. B. „Explorieren“, „Interpretieren“, „Paraphrasieren“), 


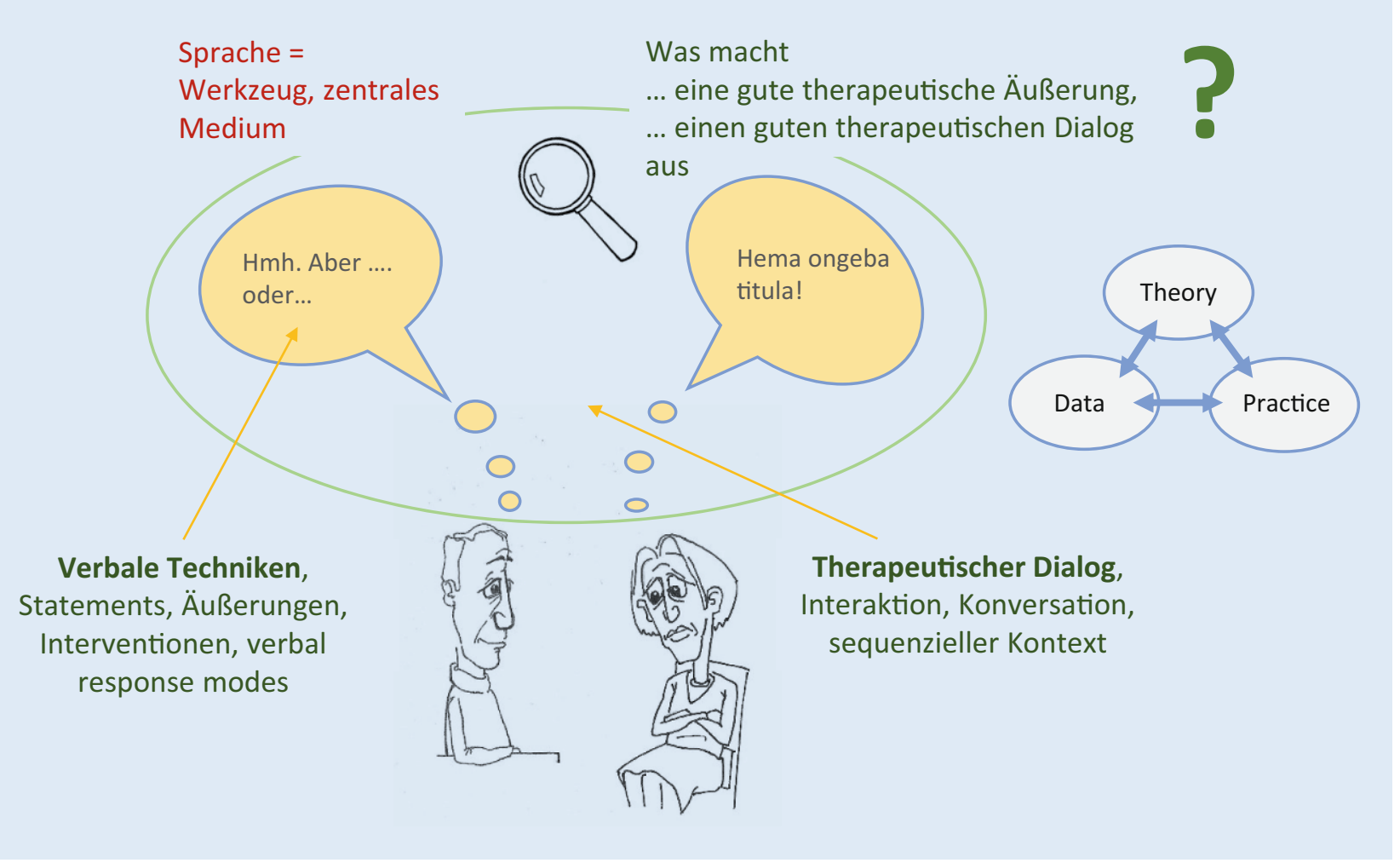

Abb. 1 A Perspektiven auf sprachliche Merkmale in derPsychotherapieforschung. (Bild „Patientin und Therapeut " mitfreundlicher Genehmigung, $\odot$ Antje Gumz/Axel Kühn, alle Rechte vorbehalten)

b) thematischer und c) zeitlicher Bezug der Äußerung (Gumz et al. 2015), der Fokus liegt also auf dem Wie von Äußerungen (Deppermann 2008, S. 9).

Bei der Analyse der Interaktion zwischen Patient und Therapeut (Fokus Dialog) stehen sprachliche Handlungen und ihr sequenzieller Kontext im Vordergrund. Die Gesprächsteilnehmer zeigen darin jeweils an, wie sie einander verstanden haben, welche Folgehandlungen sie elizitieren und ob diese ihre Erwartungen erfüllen. Zentrale therapeutische Interventionen sind Extensionen (i.e. Äußerungserweiterungen), Reformulierungen, Fragen und Interpretationen (Weiste und Peräkylä 2015). Diese heben einzelne Aspekte zur weiteren Bearbeitung hervor, sie fokussieren Aspekte subjektiven Erlebens oder setzen solche Aspekte in Zusammenhang, um so Musterhaftigkeit des Handelns und der Wahrnehmung zu verdeutlichen (Beispiele zu Forschungsergebnissen zur therapeutischen Konversation: Buchholz et al. 2022).

\section{Ziele der Arbeit}

Der Beitrag soll exemplarisch veranschaulichen, wie eine Psychotherapiesitzungssequenz mit zwei mikroanalytischen Methoden im Hinblick auf sprachliche Merkmale charakterisiert werden kann. Die Sequenz wird zunächst aus Perspektive der psychodynamischen Theorie inhaltlich interpretiert. Anschließend wird sie mithilfe einer quantitativen Methode (Psychodynamische Interventionsliste [PIL], Fokus Therapeutenäußerungen) sowie einer qualitativen Methode (Konversationsanalyse, Fokus Dialog) beurteilt.

\section{Material und Methoden}

Fokus Therapeutenäußerungen Psychodynamische Interventionsliste

Die verwendeten psychodynamischen Techniken werden mithilfe der PIL (Gumz 2018; Gumz et al. 2017, 2014) operationalisiert. Die PIL wurde mit einem „Mixedmethods"-Forschungsansatz entwickelt.
Sie umfasst 37 Kategorien verbaler Techniken, die sich ihren semantischen Merkmalen nach 3 Dimensionen zuordnen lassen: Interventionsform (24 Kategorien), thematischer Bezug (9 Kategorien) und zeitlicher Bezug (4 Kategorien). Jede Therapeutenäußerung wird separat im Hinblick darauf geratet, in welchem Ausmaß die jeweilige Kategorie zutrifft (Skala von 0 "nicht vorhanden" bis 5 "Merkmale vollständig zutreffend"). Die Kategorien und deren Kurzdefinitionen sind einsehbar unter: https://www.psychologische-hochschule. de/wp-content/uploads/2020/01/Shortdefinitions-of-PIL-categories_Gumz-etal..pdf.

Für Auswertungen auf Ebene ganzer Sitzungen können die Daten der Mikroebene (therapeutische Äußerungen) aggregiert werden: Das prozentuale Gewicht je Kategorie kann berechnet werden, um das Ausmaß zu bestimmen, in dem die Kategorien in einer Sitzung verwendet wurden (Quotient aus der Summe der Werte der jeweiligen Kategorie und der Summe der Werte aller Kategorien pro Dimension [Form, thematischer, zeitlicher Bezug]). 


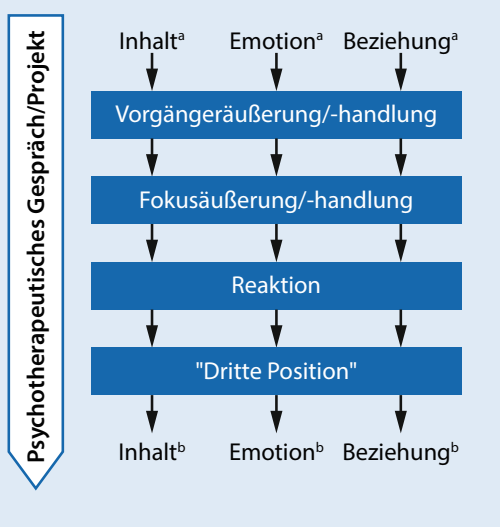

Abb. $2 \Delta$ Basissequenzmuster. ${ }^{\text {a }}$ Status vor der Sequenz, ${ }^{\text {b }}$ Status nach der Sequenz. (Nach Peräkylä 2019)

Die Interrater-Reliabilität und konvergente Validität der PIL wurden belegt ( $n=8717$ therapeutische Äußerungen; $n=38$ Sitzungen; Gumz et al. 2017). Der Intraklassen-Korrelation (ICC) betrug im Mittel über alle PIL-Kategorien 0,80, wenn ganze Sitzungen beurteilt wurden ( $n=38$ Sitzungen) und 0,68 auf Ebene der einzelnen therapeutischen Äußerungen ( $n=8717$ therapeutische Äußerungen; ICC der Kategorien, die im Sitzungsausschnitt in vorliegendem Beitrag als mindestens „kaum/wenig/gering vorhanden“ geratet wurden: 0,65-0,89). Für die Anwendung der PIL ist somit generell ein geschulter Rater ausreichend (Gumz et al. 2017). Das Rating der Sitzungssequenz in vorliegendem Beitrag wurde von zwei geschulten unabhängigen Ratern durchgeführt. Deren Werte wurden gemittelt.

\section{Fokus Dialog - Konversationsanalyse}

Die linguistische Gesprächsanalyse untersucht Gespräche nach den sprachlichen Aktivitäten der Gesprächsteilnehmer. Diese Aktivitäten werden in ihrem sequenziellen Kontext betrachtet: Therapeut $(\mathrm{T})$ und Patient $(P)$ müssen sich stets zu den Äußerungen und Interventionen ihrer Gesprächspartner verhalten. Durch Bestätigung, Widerspruch oder auch Nichtbeachtung generieren sie Intersubjektivität, gemeinsames Wissen, das sich im Verlauf immer weiter anreichert und womit Handlungsziele erreicht werden können.

Im Basissequenzmuster (• Abb. 2) zeigen sich auf kleinem Raum schon Ver-

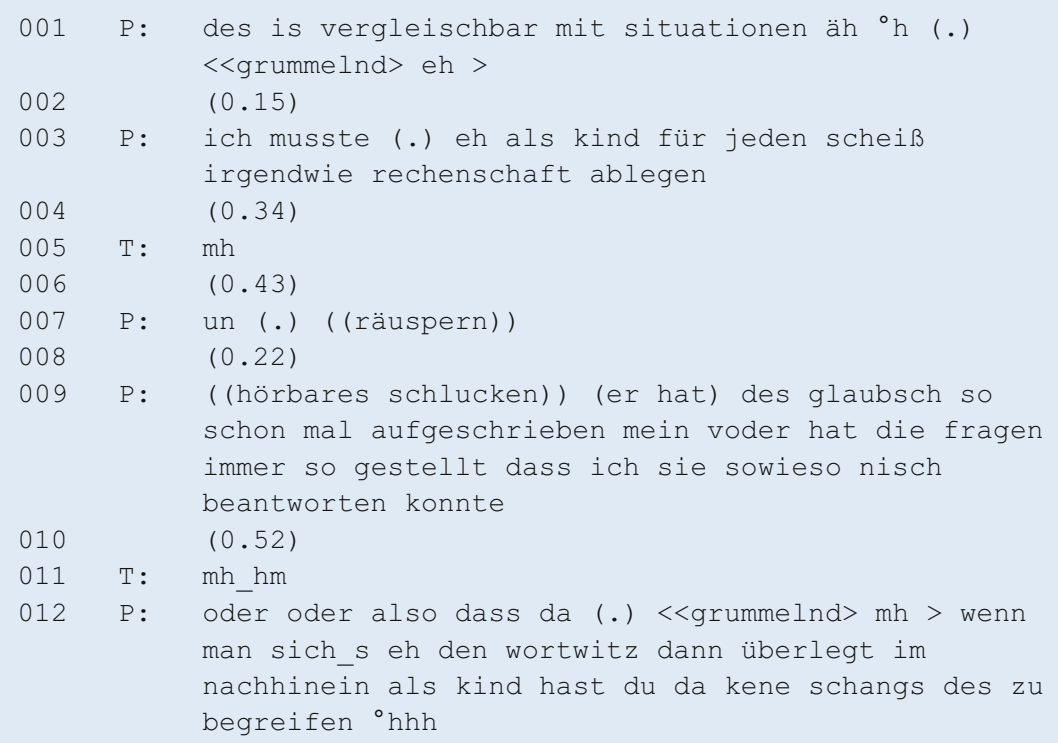

Abb. $3 \Delta$ Basistranskript nach dem Gesprächsanalytischen Transkriptionssystem

änderungen hinsichtlich Emotion und Beziehung, und auch wie Themen bearbeitet werden, sich entwickeln sowie welche verbalen und paraverbalen Merkmale Formulierungen haben. Sequenzialität wird zum Vehikel von Veränderung von Therapie.

\section{Sitzungsausschnitt}

Der betrachtete Ausschnitt entstammt der 30. Sitzung einer analytischen Therapie (durchgeführt im Sitzen) mit einem 31-jährigen Patienten (Gumz et al. 2008; Gumz und Storck 2018). Aus Datenschutzgründen wurden Details so weit verfremdet, dass Rückschlüsse auf die Person des Patienten nicht möglich sind. Herr A. kam mit rezidivierenden Panikattacken und einer depressiven Verstimmung in die Behandlung. Er litt unter häufigen Albträumen (Szenen aus dem Berufsalltag, in denen er sich gegen Kollegen wehrt und diese mit einem Knüppel angreift). Biografisch berichtete er über einen Vater, der den drei Kindern und der Mutter gegenüber regelmäßig körperlich gewalttätig wurde. Die Übergriffe waren sadistisch gefärbt und erfolgten unberechenbar und willkürlich. Die Mutter beschrieb er als nichtschützend, sie führte ihre Kinder dem Vater vor. Sie war emotional nicht verfügbar und drohte in belastenden Situationen damit sich umzubringen.

\section{Transkription des Sitzungs- ausschnitts}

Mikroanalytische Auswertungen erfordern die Einhaltung vorgegebener wissenschaftlicher Standards bei der Transkription. Für die Analysen mit der PIL wird gemäß den Transkriptionsregeln von Mergenthaler (1992) transkribiert. Für die Konversationsanalyse gelten noch stärker detaillierte Transkriptionsregeln. Neben den Äußerungen werden auch das Einund Ausatmen, Pausen, Zeilentrennung nach Intonationsphrasen, Überlappungen etc. notiert, um die Formulierungsdynamik und die Feinabstimmung der Gesprächsteilnehmer zu erfassen. Aus Platzgründen haben wir die ausführlichen Transkriptionen im Folgenden auf den Wortlaut der zentralen Aussagen hin zusammengefasst und die Sitzungssequenz somit stark verkürzt verschriftlicht. In - Abb. 3 ist anhand der ersten Patientenäußerung veranschaulicht, wie die ausführliche Transkription gemäß dem Gesprächsanalytischen Transkriptionssystem 2 (GAT2; Selting et al. 2009) erfolgt.

In der in 0 Abb. 3 gezeigten detaillierten Transkription werden die Formulierungsdynamik und die Interaktion der beiden Gesprächsteilnehmer deutlich. Im Folgenden wird der Sitzungsausschnitt dargestellt, der den exemplarischen Mikroana- 
Tab. 1 Sitzungsausschnitt: „verbale Prügel“

P1 $\quad$ Es ist vergleichbar mit Situationen, ich musste als Kind für jeden Scheiß Rechenschaft ablegen, und mein Vater hat die Fragen sowieso immer so gestellt, dass ich sie sowieso nicht beantworten kann, also wenn man sich den Wortwitz mal überlegt, als Kind haste da sowieso keine Chance, das zu begreifen

\begin{tabular}{l|l}
\hline T1 & Absichtlich hat er das dann ...
\end{tabular}

P2 $\quad$ Ich würde inm hier Absicht vorwerfen. Und ähm ... bzw. so fasse ich das, im Grunde genommen, auch hier sehr oft auf, dass ich Ihnen hier verpflichtet bin, Rechenschaft abzulegen

T2 Und erleben Sie mich auch so willkürlich und sadistisch wie Ihren Vater, dass ich Sie in so ausweglose Situationen bringe und sogar mit Absicht?

\begin{tabular}{l|l|}
\hline P3 & Durchaus ja \\
\hline T3 & Wann haben Sie das so erlebt? \\
\hline P4 & $\begin{array}{l}\text { Na, eigentlich immer in den Situationen, wo ich nicht weiterkomme, dass ich da massiv } \\
\text { unter Druck bin. Aber sadistisch ist vielleicht übertrieben. Weil, so massiv ist es in unserer } \\
\text { Beziehung ja nicht in der Art }\end{array}$ \\
\hline T4 & Also erleben Sie es so verdünnt \\
\hline P5 & $\begin{array}{l}\text { Hm. Naja und, ähm, was mein Vater, im Grunde genommen, in Extremzuständen ge- } \\
\text { macht hat, hat meine Mutter verdünnt weitergemacht oder auch meine Oma. Die hat } \\
\text { mich zwar vor meiner Mutter wiederum beschützt, aber mich immer wieder bloßgestellt, } \\
\text { wenn sie der Meinung war ... das ist so eine Situation, wo man sich nie sicher war, bei } \\
\text { wem ist man denn hier eigentlich geborgen }\end{array}$ \\
\hline T5 & Schlimm \\
\hline P Patient, TTherapeutin \\
\hline
\end{tabular}

lysen mit Fokus auf die verbalen Inhalte unterzogen wurde (• Tab. 1).

\section{Ergebnisse}

\section{Sitzungsausschnitt aus Perspektive der psychodynamischen Theorie}

Zu Beginn der Sitzungssequenz formuliert $\mathrm{P}$ einen Übertragungsaspekt. $\mathrm{Er}$ vergleicht die therapeutische Beziehung mit der Beziehung zum Vater, in der er sich hilflos und ohnmächtig fühlte. Ein Teil der Übertragung ist somit bewusstseinsfähig geworden (P1). Anschließend konkretisiert $\mathrm{T}$ die Übertragungssituation: Sie fragt direkt nach, ob $\mathrm{P}$ sie als willkürlich und sadistisch wie den Vater erlebe (T2). Der P bestätigt dies (P3), relativiert es aber kurz darauf und betont einen Aspekt der Realbeziehung - so massiv sei es nicht (P4). Damit nimmt er gleichzeitig auch ein Stück Spannung aus der Übertragungsbeziehung. Interessant ist, dass P kurz darauf auch die Mutter zum Thema macht und sie durch den Begriff „verdünnt" der T noch stärker gleichsetzt als den Vater. Diese Mutterübertragung scheint dem $P$ weniger bewusst zu sein. Sie wird auch von T nicht aufgegriffen. Die T stellt sich empathisch an die Seite des $P$ und interveniert supportiv (T5).
Mikroanalyse Fokus Therapeutenäußerungen - Psychodynamische Interventionsliste

Es werden nur jene PIL-Kategorien berichtet, die beim Rating mit mindestens 1 (, in geringem Ausmaß vorhanden“) bewertet wurden. Der Mittelwert aus zwei unabhängigen Ratings je Kategorie wird in Klammern angegeben.

T1: wurde geratet als formale Kategorie „Bedeutung hinzufügen“ (5). Die Kategorie ist definiert als "T gibt den vom $\mathrm{P}$ beschriebenen Inhalten eine neue Bedeutung, stellt sie in einen bisher nichtformulierten Zusammenhang oder fügt sie in einen neuen Kontext ein." Die Aussage nimmt thematisch Bezug auf den „Vater" als Primärobjekt (5). Primärobjekte sind definiert als Objekte (Personen), die die Persönlichkeitsstruktur in der Kindheit oder Jugend geprägt haben. Sie werden auch kodiert, wenn es um diese Objekte in der Gegenwart geht. Zeitlich bezieht sich die Intervention auf die "Vergangenheit" des P (zeitlicher Bezug, (5), definiert als Kindheit oder Jugend des $\mathrm{P}$ ).

T2: wurde dreiformalen Kategorien zugeordnet: „Bedeutung hinzufügen“ $(4,5)$, „Wiederholen, Umschreiben, Zusammenfassen" (4,5; Definition "Inhalte zuvor formulierter Äußerungen des P werden wie- derholt, umschrieben, paraphrasiert, differenzierend zusammengefasst oder auf den Punkt gebracht."). In gewissem Ausmaß wurden auch die Kategorien „Ins Hier und Jetzt der therapeutischen Beziehung holen“ (1,5; Definition: „T verbindet die Patientenaussagen direkt mit der therapeutischen Beziehung") sowie "parallele Andere wie Eltern" $(4,5)$ als zutreffend beurteilt (Definition: Zusammenhang zwischen einer Wahrnehmung des Verhaltens eines oder mehrerer Aktualobjekte, z.B. Partner, Therapeut, und dem Erleben einer primären Bezugsperson, z. B. Mutter). Allerdings hat der $P$ in seiner Äußerung davor diese Parallele primär selbst hergestellt und das Thema ins Hier und Jetzt der therapeutischen Beziehung geholt. Thematischer Bezug: "Vater" als Primärobjekt (5) und "Therapeutin" (5). Zeitlicher Bezug: "Realität" (5) und „Vergangenheit" $(4,5)$.

T3: wurde der formalen Kategorie „Explorieren" (5) zugeordnet (definiert als „Einholen neuer Informationen, mit oder ohne direkte Frage"). Thematischer Bezug: Primärobjekt "Vater" (4) und "Therapeutin“ (5). Zeitlicher Bezug: „Realität“ (5) und "Vergangenheit" (4).

T4: wurde der formalen Kategorie „Wiederholen, Umschreiben, Zusammenfas-

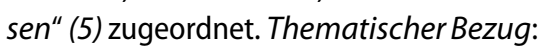
Primärobjekt "Vater" (4) und "Therapeutin“ (5). Zeitlicher Bezug: „Realität” (5) und "Vergangenheit" (4).

T5: wurde der formalen Kategorie „emotionale Anteilnahme“ (5) zugeordnet (Definition: verbal formulierte Reaktion auf Ps Äußerungen [Mitgefühl, Trost, emotionale Anteilnahme am Erleben des P]). Thematischer Bezug: "Vater", "Mutter" und „anderes Primärobjekt" (je 5). Zeitlicher Bezug: "Vergangenheit" (5).

Prozentuales Gewicht der in diesem Sitzungsausschnitt verwendeten Kategorien:

- Formale Kategorien: „Bedeutung hinzufügen“ 24,7; „Wiederholen, Umschreiben, Zusammenfassen" 23,5; „Explorieren“ 12,4; emotionale Anteilnahme 12,4; „parallele Andere

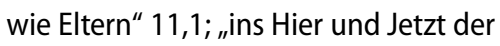
therapeutischen Beziehung holen" 3,7,

- thematischer Bezug: „Vater" 47,9; "Therapeutin" 31,2; „Mutter" 10,4; „anderes Primärobjekt" 10,4,

- zeitlicher Bezug: „Vergangenheit“ 60,0; „Realität" 40,0. 


\section{Mikroanalyse Fokus Dialog -} Konversationsanalyse

Der $\mathrm{P}$ vergleicht die aktuelle Therapiesituation mit früheren Interaktionserfahrungen, die ihm unlösbare Interaktionszwänge auferlegt hatten: Er bekommt Aufgaben vom Vater so gestellt, dass sie nicht bearbeitbar sind. Dies wird als unausweichlich bezeichnet und sprachlich-formulatorisch hochgestuft, u.a. durch den dreimaligen Gebrauch des Unveränderbarkeit signalisierenden Ausdrucks "sowieso“ (P1). Die faktizistische Darstellung Ps zum Handeln des Vaters wird von T dann - im indikativischen, also Behauptungsmodus auf die Ebene des intentionalen Handelns (",absichtlich") gehoben (T1). Ein solcher Ebenenwechsel ist typisch für Therapiegespräche, da P oft auf der Ebene faktischer Beschreibungen bleiben, $T$ dagegen auf die Erlebensebene fokussieren, subjektive Aspekte hervorheben, fokussieren oder in Beziehung setzen (qua "highlighting, rephrasing, relocating activities"). Reformulierungen dieser Art gehören zu den häufigsten therapeutischen Aktivitäten (Weiste und Peräkylä 2015).

Der Ebenenwechsel im Gesprächsausschnitt kann $\mathrm{P}$ aber auch entlasten: Diese Situationen seien durch Böswilligkeit des Vaters und nicht durch eigenes Unvermögen entstanden. Der $P$ bestätigt dies, schwächt jedoch den Vorwurf durch den Gebrauch des Konjunktivs ab. AnschlieBend charakterisiert er die Therapiesituation als direkt vergleichbar. Die beschriebenen Situationskonstellationen in Vergangenheit und Gegenwart sollen damit intersubjektiv als Voraussetzung weiteren (Be-)Handelns gelten.

Mit der nächsten Intervention (T2) pointiert T verschiedene Charakterisierungen ins Extreme: „willkürlich ... sadistisch ... ausweglos ... mit Absicht". Solche "extreme case formulations" (Pomerantz 1986) dienen dazu, eine vertiefte, ggf. auch zurückweisende Auseinandersetzung anzustoßen. Hier im Fallbeispiel weist $\mathrm{P}$ das Angebot allerdings nicht zurück. Er bestätigt die Charakterisierungen dezidiert und eindeutig mit "durchaus ja" (P3).

Die T fragt daraufhin nach Beispielen aus dem bisherigen Therapieprozess (T3). Eine solche Beispielnachfrage soll stets
Situationsbeschreibungen anregen, in denen viele Aspekte der Wahrnehmung, Bewertung und des Verhaltens Ps sichtbar werden (Spranz-Fogasy et al. 2020). Der $P$ antwortet hier aber zunächst eher ausweichend und allgemein, dennoch qua „massiv unter Druck“ formulatorisch pointiert, was er gleich darauf relativiert, diese Rücknahme aber selbst wiederum relativiert (P4). Der P beschreibt demnach auch die Therapiesituation als stark Druck ausübend. Die T reformuliert dies als "verdünnt" (T4), damit aber immer noch als markantes inneres Erleben Ps.

Aufdieser Grundlage präsentiertP dann ein komplexes Familienbild mit absteigend starker Zuschreibung gegen ihn gerichteten Handelns: Vater - Mutter - Oma und der sich aus dieser Konstellation ergebenden kompletten Unsicherheit anderen Menschen gegenüber (P5). Auffällig sind dabei der Fokuswechsel auf die Mutter und die Oma sowie die Übernahme der verbalen Zuschreibung von „verdünnt", hier v.a. an die Mutter als Täterin. Den Ausdruck "verdünnt" hatte T vorher der Therapiesituation bzw. sich selbst zugeordnet (T4). Die T kommentiert diese Beschreibung mit dem Ausdruck "schlimm" und stellt sich damit als mitfühlend und unterstützend dar (T5).

\section{Diskussion}

Sprache ist das zentrale Agens in Psychotherapien. Mit ihr werden psychische Probleme in den psychischen Binnenraum überführt, integriert und behandelbar gemacht.

Zur Untersuchung der Sprache von Therapeuten stehen unterschiedliche qualitative Verfahren (Voutilainen und Peräkylä 2014) sowie eine Reihe von beobachterbasierten und computerautomatisierten Messinstrumenten zur Verfügung (Steinert et al. 2022; Kadur et al. 2020; Gumz et al. 2015). Ziel des vorliegenden Beitrags war, eine quantitative und eine qualitative Methode exemplarisch vorzustellen.

Die analysierte Sitzungssequenz zeigt die interaktionale Kokonstruktion einer komplexen konkreten Erkenntnis. Thematisch geht es um die Ausdeutung der Therapiesituation als vergleichbar mit früheren Erfahrungen des Patienten. Die
Therapeutin zergliedert die Real- und Übertragungssituation(en). Der Patient überträgt in dieser und anderen Sitzungen sowohl Aspekte der Beziehung zum Vater als auch der Beziehung zur Mutter. Er erlebt die Therapeutin ähnlich unberechenbar, willkürlich und sadistisch. Er erwartet weder Schutz noch emotionale Zuwendung, sondern fühlt sich verfolgt und ohnmächtig ausgeliefert - stets in Erwartung, vorgeführt und gedemütigt zu werden. Die Therapeutin verständigt sich gemeinsam mit dem Patienten aus einer abstrakten Beobachterperspektive heraus darüber, inwieweit die gemeinsam konstruierte aktuelle Beziehung durch Beziehungserfahrungen mit den Eltern des Patienten geprägt wird. In der analysierten Sitzungssequenz und im weiteren Verlauf der Sitzung (Gumz et al. 2008; Gumz und Storck 2018) gelingt es der Therapeutin und dem Patienten sukzessive, die Erlebens- und Verhaltensmuster des Patienten zu reflektieren: in Bezug auf die Vergangenheit, die therapeutische Situation und die aktuelle "Außenwelt" des Patienten. Begleitend wird der Patient von schuldhafter Mitverantwortung und eigenem Versagen entlastet, und es werden machbare Lösungsschritte formuliert („Vielleicht wäre es ja der erste Schritt zu sagen, jetzt komme ich hier wieder unter Druck. Damit wir dann gemeinsam prüfen, was da passiert" [im späteren Sitzungsverlauf, hier nicht abgebildet]).

Der Sitzungsausschnitt zeichnet sich durch lenkende Aktivitäten der Therapeutin aus, auf die der Patient aber stets sehr kooperativ und reflektiert reagiert. Das Thema wird Schritt für Schritt in seiner Breite und Tiefe ausgeleuchtet. Der Patient wird immer wieder an Punkte geführt, die er explizit bestätigen kann, wodurch die Ergebnisse der Aushandlung jeweils in den intersubjektiven Wissensbestand eingebaut werden können (,ich würde ihm hier Absicht vorwerfen“, "durchaus ja“, bis hin zu einem abschließenden emphatischen "so isses!" [im späteren Sitzungsverlauf, hier nicht abgebildet; Gumz et al. 2008; Gumz und Storck 2018]).

Formulatorisch finden sich viele Wiederaufnahmen und Übernahmen von Ausdrücken ("Druck“, "hier/jetzt", „Rechenschaft ablegen“, ",verdünnt" etc.), was zeigt, dass die Beteiligten nahe am Thema, 
am Hier und Jetzt der Therapiesituation, an ihren eigenen Aussagen und denen des Gesprächspartners bzw. der - partnerin bleiben.

Durch die gesprächsanalytische Mikroanalyse wird deutlich, wie die Beteiligten in ständiger wechselseitiger, sequenziell organisierter Abstimmung den Kern eines traumatischen Geschehens und seinen Einfluss auf das Erleben und Verhalten des Patienten in seiner Lebenswelt und in der Therapiesituation herausschälen. Dies bildet die Grundlage für Veränderung, die die zentrale Rationale therapeutischen Handelns ist (Weiste und Peräkylä 2015).

Die linguistische Gesprächsanalyse ist ein wertvoller Ansatz, Veränderungsprozesse im Gesprächsprozess zu identifizieren und therapeutisch nutzbar zu machen. Die Gesprächssequenzen werden Schrittfür-Schritt im Detail beobachtet. Anhand des sprachlichen Austauschs wird sichtbar, wie sich die Interaktion entfaltet und sich Inhalte, Emotionen und Beziehungen verändern. Intersubjektivität zeigt sich darin, dass die Gesprächspartner die Äußerungen des/der Anderen bestätigen, wiederaufnehmen oder bestimmte Ausdrücke übernehmen. Therapeut und Patient versichern sich damit gegenseitig, dass sie sich richtig verstanden haben und von einer gemeinsamer Wissensbasis ausgehen, die Grundlage weiterer Schritte sein kann. Auf der sprachlichen Oberfläche wird auch sichtbar, ob Patienten "mitgehen" oder ob sie unsicher sind, sich verweigern, widersprechen oder Äußerungen der Therapeutin nicht beachten. Es kann somit sehr aufschlussreich sein, wenn Psychotherapeuten die eigene Sprache und den Dialog im Therapieprozess genau beobachten. Dies kann ermöglichen, das eigene Vorgehen bereits auf der Mikroebene des sprachlichen Austauschs anzupassen.

Die Mikroanalyse mithilfe der PIL zeigt, dass in diesem Sitzungsausschnitt überwiegend und in etwa gleichwertigem Ausmaß die verbalen Techniken „Bedeutung hinzufügen" und "Wiederholen, Umschreiben, Zusammenfassen" angewandt werden. Thematisch wird am stärksten auf den "Vater" Bezug genommen, gefolgt von der "Therapeutin“. Der zeitliche Bezug liegt schwerpunktmäßig in der "Vergangenheit". Die mithilfe der PIL vorgenommene Zuordnung zu vorgege- benen Kategorien von Techniken reduziert Information, die im Dialog und in den theoretischen Konzepten enthalten ist, zum Zwecke der Operationalisierung. Dies ermöglicht, Psychotherapieprozessforschungen durchzuführen. Die Wechselwirkungen zwischen verbalen Techniken, Therapeuten-, Patientenfaktoren und Therapieergebnis genauer zu verstehen, ist ein klinisch äußerst relevantes Forschungsanliegen. Hierzu gehört der Zusammenhang verwendeter verbaler Techniken mit dem Therapieergebnis, der Sitzungsqualität (Mikro-Outcome, z. B. therapeutische Allianz oder andere in der Sitzung verwirklichte allgemeine Wirkfaktoren) oder anderen relevanten Prozessvariablen. In diesem Kontext ist auch von hohem Interesse, zu welchem Ausmaß therapeutische Veränderung im Zusammenhang steht, mit verbalen Techniken, die nicht intendiert oder nicht theoretisch relevant sind, oder inwieweit sich unterschiedliche therapeutische Methoden im Hinblick auf die in der Praxis verwendeten Techniken unterscheiden (Gumz et al. 2015). Neben dem Einsatz in der Psychotherapieforschung bietet die PIL eine empirisch fundierte Systematik verfügbarer Interventionstypen, die konkret handwerkliches Wissen enthält. Dies ist von Nutzen für die psychotherapeutische Ausbildung.

Die Sprache ist das Werkzeug des Psychotherapeuten, und der sprachliche Dialog zwischen Patient/-in und Therapeut/-in ist in Psychotherapien zentral. Die empirische Befundlage zu dem, was auf sprachlicher Ebene im Therapieprozess geschieht, und zum Zusammenhang von Sprache mit dem Therapieergebnis oder anderen Prozessvariablen ist insgesamt noch schwach. Die bislang vorliegenden Studien sind interessant und vielversprechend. Sie zeigen, dass die Art, wie Therapeuten sprechen, wichtige klinische Erkenntnisse enthält sowie die Therapiebeziehung und das Therapieergebnis beeinflusst. Weiterführende Forschungen an der interdisziplinären Schnittstelle von Psychotherapie und Linguistik sind wünschenswert.

\section{Fazit für die Praxis}

- Linguistische Mikroanalysen von Therapiesitzungen können Dynamiken der the- rapeutischen Interaktion (ÜbertragungGegenübertragung-Muster) und Veränderungsprozesse sichtbar und nutzbar machen.

- Auf der sprachlichen Oberfläche wird sichtbar, wie die Interaktion sich entfaltet sowie Inhalte, Emotionen und Beziehungen sich verändern.

- Sichtbar wird, ob die Gesprächspartner Äußerungen bestätigen, wiederaufnehmen oder bestimmte Ausdrücke übernehmen und somit von einer gemeinsamen Wissensbasis ausgehen.

- Sichtbar wird auch, ob die Gesprächspartner unsicher sind, sich verweigern, widersprechen oder Äußerungen des/der Beteiligten nicht beachten.

- Die eigene Sprache und den Dialog im Therapieprozess genau zu beobachten, kann für den Therapeuten/die Therapeutin zu wichtigen Erkenntnissen führen und ermöglichen, das eigene Vorgehen bereits auf der Mikroebene des sprachlichen Austauschs anzupassen.

- Die Psychodynamische Interventionsliste kann für die Prozessforschung und als konkrete handwerkliche Übersicht für die psychotherapeutische Ausbildung genutzt werden.

Korrespondenzadresse

\section{Prof. Dr. Antje Gumz}

Professur für Psychosomatik und Psychotherapie, Psychologische Hochschule Berlin

Am Köllnischen Park 2, 10179 Berlin,

Deutschland

a.gumz@phb.de

Funding. Open Access funding enabled and organized by Projekt DEAL.

\section{Einhaltung ethischer Richtlinien}

Interessenkonflikt. A. Gumz und T. Spranz-Fogasy geben an, dass kein Interessenkonflikt besteht.

Alle beschriebenen Untersuchungen am Menschen oder an menschlichem Gewebe wurden mit Zustimmung der zuständigen Ethikkommission, im Einklang mit nationalem Recht sowie gemäß der Deklaration von Helsinki von 1975 (in der aktuellen, überarbeiteten Fassung) durchgeführt. Von allen beteiligten Patienten liegt eine Einverständniserklärung vor.

Open Access. Dieser Artikel wird unter der Creative Commons Namensnennung 4.0 International Lizenz veröffentlicht, welche die Nutzung, Vervielfältigung, Bearbeitung, Verbreitung und Wiedergabe in jeglichem Medium und Format erlaubt, sofern Sie den/die ursprünglichen Autor(en) und die Quelle ordnungsgemäß nennen, einen Link zur Creative Commons Lizenz beifügen und angeben, ob Änderungen vorgenommen wurden. 
Die in diesem Artikel enthaltenen Bilder und sonstiges Drittmaterial unterliegen ebenfalls der genannten Creative Commons Lizenz, sofern sich aus der Abbildungslegende nichts anderes ergibt. Sofern das betreffende Material nicht unter der genannten Creative Commons Lizenz steht und die betreffende Handlung nicht nach gesetzlichen Vorschriften erlaubt ist, ist für die oben aufgeführten Weiterverwendungen des $\mathrm{Ma}$ terials die Einwilligung des jeweiligen Rechteinhabers einzuholen.

Weitere Details zur Lizenz entnehmen Sie bitte der Lizenzinformation auf http://creativecommons.org/ licenses/by/4.0/deed.de.

\section{Literatur}

Breuer J, Freud S (2007) Studien über Hysterie. Fischer, Frankfurt/Main (1895)

Buchholz MB, Alder ML, Dreyer F, Franzen MM (2022) Sprechen und Schweigen in der Psychotherapie - Unterwegs zu einem kommunikativen "turn“. Psychotherapeut. https://doi.org/10. 1007/s00278-021-00563-w

Deppermann A (2008) Gespräche analysieren, 4. Aufl. Verlag für Sozialwissenschaften, Wiesbaden

Gelo O (2022) Computer-assisted text analysis in psychotherapy research: the therapeutic cycles. Psychotherapeut. https://doi.org/10. 1007/s00278-022-00577-y

Georgaca E, Avdi E (2009) Evaluating the talking cure: the contribution of narrative, discourse, and conversation analysis to psychotherapy assessment. Qual Res Psychol 6:233-247

Gumz A (2018) Psychodynamische Interventionstechniken. In: Gumz A, Hörz-Sagstetter S (Hrsg) Psychodynamische Psychotherapie in der Praxis. Lehrbuch. Beltz, Weinheim, S159-170

Gumz A, Storck T (2018) Übertragung und Gegenübertragung. In: Gumz A, HörzSagstetter S (Hrsg) Psychodynamische Psychotherapie in der Praxis. Beltz, Weinheim, S43-50

Gumz A, Villmann T, Bergmann B et al (2008) Übertragung. Ein attraktiver Systemzusatnd. Forum Psychoanal 24:229-245

Gumz A et al (2015) Measuring verbal psychotherapeutic techniques-a systematic review of intervention characteristics and measures. Front Psychol 6:1705

Gumz A, Horstkotte JK, Kästner D (2014) The techniques of the psychodynamic therapist. Types of verbal interventions from a theoretical and practice-oriented perspective. Z Psychosom Med Psychother 60:219-237

Gumz A, Neubauer K, Horstkotte JK, Geyer M, Löwe B, Murray AM, Kästner D (2017) A bottomup approach to assess verbal therapeutic techniques. Development of the Psychodynamic Interventions List (PIL). PLoS ONE. https://doi. org/10.1371/journal.pone.0182949

Kadur J, Lüdemann J, Andreas S (2020) Effects of the therapist's statements on the patient's outcome and the therapeuticalliance: a systematic review. Clin Psychol Psychother. https://doi.org/10. 1002/cpp.2416

Levitt HM, Motulsky SL, Wertz FJ, Morrow SL, Pontoretto JG (2017) Recommendations for designing and reviewing qualitative research in psychology: promoting methodological integrity. Qual Psychol 4:2-22

Marx C, Benecke C, Gumz A (2017) Talking cure models: a framework of analysis. Front Psychol 8:1589

\section{Linguistic characteristics of a psychotherapy session sequence from the perspective of different analytical methods}

Background: Linguistic utterances are a central medium in psychotherapy, i.e., psychotherapy essentially works through language, through talking to each other. Given the importance of linguistic exchange, it is relevant to more precisely understand the mechanisms through which language operates in psychotherapy processes. Linguistic psychotherapy research uses a variety of methods for this purpose.

Objective: This article demonstrates two microanalytical approaches as examples. Material and methods: A transcribed psychotherapy session sequence was interpreted in terms of content from the perspective of the psychodynamic theory and assessed microanalytically in terms of linguistic features using two methods. First, the verbal techniques (focus on therapist utterances) were rated using the psychodynamic intervention list (PIL). Second, a detailed conversation analysis (focus on dialogue) was conducted.

Results: Analyses using the PIL showed that "adding new meaning" and "repeating, paraphrasing, summarizing" were the techniques most frequently used in the session sequence under examination. Thematically, the focus was on the "father", followed by the "therapist". The majority of the utterances thematically referred to the past. Conversation analysis reconstructs that the shift to the experiential level brings the therapy situation itself into focus. By means of sequential action constraints, extratherapeutic constellations in the past and therapeutic present are made contrastable and intersubjectively processable.

Conclusion: To observe one's own way of speaking and the dialogue within the therapeutic process can provide revealing insights for therapists about the consequences and preconditions of their own interventions. It is worthwhile conducting research at the interdisciplinary interface of psychotherapy and linguistics.

\section{Keywords}

Linguistics - Verbal behavior - Psychotherapeutic processes - Psychodynamic intervention list . Sequence analysis

Marx C, Benecke C, Gumz A (2021) Wie kann Reden heilen? Zur Sprache in der Psychotherapie. Forum Psychoanal 37:99-115

Mergenthaler E (1992) Die Transkription von Gesprächen. Ulmer Textbank, Ulm

Peräkylä A (2019) Conversation analysis and psychotherapy: identifying transformational sequences. Res Lang Soc Interact 52(3):257-280

Pomerantz A (1986) Extreme case formulations: a way of legitimizing claims. Hum Stud 9:219-229

Selting M, Auer P, Barth-Weingarten D, Bergmann J, Bergmann P, Birkner K et al (2009) Gesprächsanalytisches Transkriptionssystem 2. Gesprachsforschung 10:353-402

Sidnell J, Stivers T(2013) The handbook of conversation analysis. Blackwell handbooks in linguistics. Wiley-Blackwell, Chichester

Spranz-Fogasy T, Graf E-M, Ehrenthal JC, Nikendei C (2020) Requesting examples in psychodiagnostic interviews: therapists' contribution to the sequential co-construction of clients' change. Commun Med 16(2):129-141

Steinert LM, Gries S, Kästner D, Molitor A, Wulf S, Gumz A (2022) Die Sprache der Psychotherapeut*innen. Ein systematisches Review zum Zusammenhang semantischer Merkmale mit Therapiebeziehung und Therapieerfolg. Psychotherapeut. https://doi.org/10.1007/s00278022-00570-5

Voutilainen L, Peräkylä A (2014) Therapeutic conversation. In: Östmann J-O, Verschueren J (Hrsg)
Handbook of pragmatics. John Benjamins, Amsterdam, S1-39

Weiste E, Peräkylä A (2015) Therapeutic discourse. In: Tracy K (Hrsg) The international encyclopedia of language and social interaction. The WileyBlackwell-ICA international encyclopedias of communication. Wiley-Blackwell, Oxford, S1-10 